\title{
The effect of curriculum organization on the acquisition of abstract declarative knowledge in computer-based instructions
}

\author{
Saleh Al-Foraih ${ }^{1} \cdot$ Kent Williams $^{2}$
}

Received: 8 December 2014/Revised: 19 May 2015/Accepted: 8 June 2015/

Published online: 25 June 2015

(C) Beijing Normal University 2015

\begin{abstract}
The United States of America has dropped behind many countries in terms of the Science and Engineering university degrees awarded since the beginning of the nineties. Multiple studies have been conducted to determine the cause of this decline in degrees awarded in order to reverse the trend in US education. The goal of these studies was to determine the proper instructional methods that facilitate the knowledge acquisition process for the student. It has been determined that no one method works for all types of curriculum, for example, methods that have been found to work effectively in curriculum that teaches procedures and physical systems often fail in curriculum that teaches abstract and conceptual content. The purpose of this study is to design an instructional method that facilitates the teaching of abstract knowledge in the Industrial Engineering curriculum, and to demonstrate its effectiveness through empirical research. An experiment including 72 undergraduate students was conducted to determine the best method of acquiring abstract knowledge. All students were presented with the same abstract knowledge but presented in different types of organization. These organization types consisted of a bottom-up moving from specific to general information, a top-down moving from general to more specific information, and Unorganized random presentation of topics. Another factor that was also introduced is graphing, which is a method that is believe to improve the learning process. The experiment was completed in 8 weeks and data were gathered and analyzed. The results strongly suggest that abstract knowledge acquisition, as it relates to Industrial Engineering concepts, is greatly improved when the knowledge is presented in a bottom-up hierarchical fashion. On the other hand, neither graphing nor the top-down or unorganized conditions affect learning in these novice students. The understanding of what facilitate learning is
\end{abstract}

Saleh Al-Foraih

salforaih@hotmail.com

1 King Abdulaziz Military Academy, Riyadh, Saudi Arabia

2 University of Central Florida, Orlando, USA 
very critical to how best to deliver content to students, especially in today's digital world. For example, in an online learning environment, an intelligent tutoring software can be used to automatically load different organizational structure of the same content based on the student response to the instruction. The same software can load different organizations of the content to personalize the learning to different students, meaning that if a student has demonstrated a learning behavior that suggests his content needs to be presented in a bottom-up fashion, while another student may demonstrate a different behavior, which would suggest his content to be presented in top-down fashion. Therefore, the curriculum can be created once but presented in different hierarchies to meet the students' needs.

Keywords Abstract knowledge - Computer-based instruction · Hierarchical knowledge organization - Graphical knowledge representation

\section{Introduction}

The United States of America has dropped behind Europe and Asia in terms of the Science and Engineering (S\&E) university degrees awarded since the beginning of the nineties (National Science Board (NSB) and National Science Foundation (NSF), 2002). Even in higher education programs, Europe has produced far more S\&E doctoral degrees $(54,000)$ than the United States $(26,000)$ (National Science Board (NSB) and National Science Foundation (NSF), 2002). Multiple studies have been conducted to determine the cause of this decline in degrees awarded in order to reverse the trend in US education. The goal of these studies was to determine instructional methods to facilitate the knowledge acquisition process for the student. It has been determined that no one method works for all types of curriculum, for example, methods that have been found to work effectively in curriculum that teaches procedures and physical systems often fail in curriculum that teaches abstract and conceptual content.

There is clearly a gap when it comes to teaching conceptual and abstract knowledge. The goal of this research is to design an instructional method that facilitates teaching of abstract knowledge, and to demonstrate its effectiveness through empirical research. The understanding of what facilitate learning is very critical on how to best deliver content to students, especially in today's digital world. For example, in an online learning environment, an intelligent tutoring software can be used to automatically personalize the curriculum for each student based on their capabilities and on the type of knowledge to be taught.

The hypothesis guiding the present research is that instructional strategies which stimulate students to generate graphical organizations of complex concepts will improve learning of abstractions.

\section{Literature review}

As early as Miller (1956), Knowledge organization has been the topic of considerable research for many years where the concept of chunking proposed the notion of a hierarchical organization of information. Miller (1956) defined 
chunking when elements of information are grouped and stored as a hierarchy, and as a result facilitates efficient memory storage.

Three years earlier, Bousfield (1953) defined clustering as a consequence of organization in thinking and recall. Therefore, the quantification of clustering provides us with information on the nature of organization as it operates in higher mental processes. Bousfield (1953) concluded from his experiment that during recall, subjects have a tendency to group the items in clusters containing members of the same general category, which implies the operation of an organizing tendency.

To determine the effect of organization on recall, Bower et al. (1969) conducted an experiment on four groups that were presented with categorically organized hierarchical trees of common words, representing minerals, animals, transportation, and clothing. Four other groups were presented with the same words, but in a randomly organized tree. The results indicated that the groups presented with the categorically organized trees outperformed the groups presented with the randomly organized trees by a vast advantage during recall tests.

To determine the effect of textual information organization on the human processing of information, Frase $(1970,1975)$ conducted multiple studies that altered the order of the sentences in a text. The data confirmed that the sequence of verbal information can determine the way in which information is stored, and therefore alter the subjects' ability to produce new combinations of that information at a later time. Frase (1970) concluded that text that is organized in a logical order provides for better recall on the part of subjects than randomly organized text. Furthermore, Frase (1975), based on other experiments, has strengthened his conclusion that the organizational characteristics of text can influence the performance of subjects on recall, and clearly emphasized the importance of organization in verbal learning.

Simon (1981) also emphasized the importance of the organization of information. He defined a hierarchy as a system that is composed of interrelated subsystems, which are in turn hierarchic in structure until they reach some lowest level of elementary subsystem. For example, when a subject is asked to draw a human face, the subject will almost always proceed in a hierarchical fashion. The subject will sketch the face first, and then he/she will add major features such as eyes, nose, mouth, ear, and hair. If the subject is asked to elaborate, he/she will continue drawing by adding details such as pupils, eyelids, lashes, and so on, until he/she reaches the limits of his anatomical knowledge. This demonstrates that knowledge about the human face is arranged in memory in a hierarchical fashion. Simon (1981) proposed that one path to the construction of a nontrivial theory of complex systems is by way of a hierarchy. He claimed that, empirically, a large portion of the complex systems we observe in nature exhibit a hierarchic structure.

Shavelson (1972) and Gagne and Briggs (1974) also identified the importance of hierarchy in sequencing and representing knowledge. While Shavelson believes that knowledge is represented from the top downward, Gagne and Briggs assumes that cognitive structure is organized from the bottom upward, and that Bottom-Up structure seems the best content organization in terms of learning abstract knowledge as well as other forms of knowledge.

Simon (1981) also argued that, by representing complex systems as hierarchical decompositions, little to no information is lost in the properties of the system. This is referred to as near decomposability as he claims that in hierarchic systems, we can 
distinguish between the interaction among subsystems on one hand, and the interactions within subsystems on the other hand. The interactions at different levels will differ depending on where the sources are in the hierarchy. For example, in a formal organization, two employees in the same department will interact more than two employees from different departments.

Complex systems were also the focus of Miyake (1986), who conducted a study to determine how people organize and communicate their understanding of how a device works. Multiple pairs of individuals, with different levels of understanding of the device, were observed communicating. Miyake (1986) found that individuals communicated how the device worked using what Miyake calls a function-mechanism hierarchy, which is made up of several levels of abstraction, each describing a different level of functions and mechanisms. Functions describe what happens at each level, while mechanisms describe how it happens. Miyake's (1986) research showed that people go through an iterative process as they explain a device, going from a high level to the lowest level of abstraction, demonstrating the existence of a hierarchical structure.

This hierarchical organization of information was also found by Anderson's (1993) research. He discussed that a feature of chunks is that they can enter into hierarchical organizations, such as chunks within chunks. For example, the string of letters D Y J H Q G W, might be organized as the chunks DY JHQ GW. Bower et al. (1969) were able to show hierarchical organization of elements in up to four levels when describing mineral elements.

Anderson (1993) described the existence of hierarchical goal structures when a person deals with problems solving. A person would solve the sub goals working their way up to the main goal in order to solve the main problem. For example, in order to be able to take his child to preschool, one may need to repair his car first. Therefore, in order to repair his car, one may need to contact an auto repair shop initially. These various goals, would be ordered on a stack, with contacting the repair shop on top of the stack. This is a Last-In-First-Out (LIFO) stack, where the last sub goal one has put on the stack is the goal one is currently focused on.

Anderson et al. (1993) undertook a geometry tutoring project that focused on instruction of traditional Euclidean proof skills. The student interacts with the computerized geometry tutor using a series of proof graphs and diagrams, to help them understand and solve sub goals, which leads them to understanding the main problem. This has been defined as reification, because the proof graphs illustrate the concrete abstract features of problem solving in geometry. These features, for which students have a great deal of difficulty, are the logical relationships among the premises and conclusions, and the search process by which one hunts for the correct proof. Students have reported that the graphical features of the proof graphs are more helpful than the traditional textual two-column proof form. Scheines and Sieg (1994) used such representations for proofs in formal logic. They found evidence that such graphical representations help problem solving even without a tutor.

In the theory of problem solving, Novick and Bassock (2005) described that two phenomenon take place during the problem solving process: representation of the problem, and solution generation. They also described that the representation may happen through either of two mechanisms, which are constraint relaxation or chunk decomposition. Constraint relaxation involves deactivating some knowledge element 
that has constrained the operators being considered, thereby allowing application of new operators. Constraint relaxation is often used where finding a solution to the original problem is prohibitively expensive or infeasible. For example, during a complex maze problem, constraint relaxation can be used to speed up the solution time. As an example where constraint relaxation is obvious, is when allowing firefighters to break through a door in order to get inside a burning house. The normal constraint would have been to unlock the door with a key to enter, but in order to solve the problem of entering the house as quick as possible, the later constraint has been relaxed to allow for forced entry.

The mechanism (chunk decomposition) involves inheriting the links that bond components of a meaningful unit in the problem. This is a clear indication that problem representation is in hierarchical form, by combining meaningful components (chunks) together with bonds to describe the state of the problem at a given time. For example, in the burning house, the firefighter can break the problem of "entering the house as quickly as possible" into multiple components. A house has multiple entry points such as windows, front door, back door, and garage door, while each requires a specific way to open. This decomposition will help the firefighter determine the best course of action to enter the house based on past experiences and the state of the art at the time.

Williams and Lopez (in press) conducted a study to determine the effect of the organization of technical curriculum for the acquisition of declarative knowledge. It was found that the group presented with the top-down organization acquired more knowledge by a factor of six versus the bottom-up group during the first trials. The top-down group also continued to outperform the bottom-up group across all trials.

Williams et al. (in progress) extended the above research to examine the top-down and bottom-up approaches when it applies to knowledge of abstract concepts and propositions, as will be referred to as "abstract" knowledge. Williams et al. (in progress) did not find any significant difference between the different groups when the curriculum relates to abstract knowledge. This finding was explained by the fact that abstract concept hierarchies have no obvious real-world representation, but have meaning only to the expert with extensive knowledge of the domain in question. Therefore, students, naïve of the terms of an abstract concept hierarchy would find it difficult to identify the explicit structure of the hierarchy. In the words of Anderson et al. (1993), this type of information is not readily reified into an abstraction hierarchy as is how-it-works knowledge, which is spatial in nature, and how-to-do-it knowledge, which is temporal in nature. Complex systems (how-it-works knowledge) are physical in nature and can readily be decomposed as indicated by Simon (1981). Problem solving is temporal in nature requiring accomplishment of sequence of how-to-do-it steps or sub goals, as indicated by Anderson (1993). As humans we have become exposed to these kinds of concrete representations which are reified and easy to relate to. Abstractions on the other hand are manufactured by humans who have considerable experience in a domain and are not readily reified.

From the literature review, it appears that providing abstract information in a hierarchical form will take advantage of the two mechanisms to support learning of abstract knowledge. The first mechanism is to stimulate greater cognitive activity by having students generate graphical representations which require students to think about relations between different levels of content regarding an abstract topic. The second mechanism takes advantage of the human's inherent capability to form hierarchical relations, which requires the cognitive system to be more engaged. The 
result is expected to improve reification of abstract knowledge and thus accelerate the learning experience.

\section{Methodology}

To investigate the above hypothesis, an experiment was conducted with seventy two, 72, undergraduate students. Participation in the experiment is open to all students, regardless of age, race, gender, or nation of origin. During the experiment, participants were required to read material based on abstract content covering concepts in Industrial Engineering (IE). The content used in this experiment was taken from a chapter in an introductory course in IE. The organization of the existing chapter contents was linear in nature with topics sequenced as created by the original author. The chapter was reorganized into a top-down framework (see below) (named top-down content). The second bottom-up set (See below) (named bottom-up content) was generated simply by reversing the order of presentation of the material created for the top-down version. The third version (named unorganized content) was generated by assigning a number to each topic in the top-down version. Numbers were then drawn from a random number table to determine the positioning of a topic in the text with the restriction that no sequence of any two numbers in either an ascending or descending order could occur. This version then was a simple linear sequencing of topics without any inherent organization. The following is the outline for the top-down and bottomup contents. They are presented side by side to show the ordering process.

Top-down content outline

The new role of the Industrial Engineer and productivity

Stages of development of the IE

Stage one: the analytic stage

Stage two: the problem solving stage

Stage three: the client centered stage

The role of the IE as consultant for change

Promoting client change

Promoting client change

The role of the IE as consultant and manager of performance improvement

Performance improvement planning process

Nominal group technique-team efforts

Productivity

Worker productivity/efficiency

Process productivity/effectiveness

Direct cost

Non-direct labor/indirect cost

\section{Bottom-up content outline}

The new role of the Industrial Engineer and productivity

Productivity

Non-direct labor/indirect cost

Direct cost

Process productivity/effectiveness

Worker productivity/efficiency

The role of the IE as consultant and manager of performance improvement

Nominal group technique-team efforts

Performance improvement planning process

The role of the IE as consultant for change

The client change process

Promoting client change

Stages of development of the IE

Stage one: the analytic stage

Stage two: the problem solving stage

Stage three: the client centered stage 
The students were divided into six groups of twelve participants to represent six distinct conditions in this experiment. These groups are divided into a control set and an experimental set. The control set contains three groups labeled "Top-Down Control Group," "Bottom-Up Control Group," and "Unorganized Control Group." These groups will respectively be subjected to content in a "Top-Down Content," "Bottom-up Content," and "Unorganized Content." The experimental set contains three groups labeled "Top-Down Experimental Group," "Bottom-Up Experimental Group," and "Unorganized Experimental Group." These experimental groups will also be, respectively, subjected to "Top-Down Content," "Bottom-up Content," and "Unorganized Content," but will be required to draw a graphical representation of the their understanding of the material prior to taking posttests. The participants are randomly assigned to ensure that each participant had an equal chance of being assigned to any one of the six different conditions. Because of the abstract nature of the content, it is believed that some participants may be unmotivated. In order to account for this factor, each participant took a motivation screening test prior to starting the experiment. Participants assigned to the graphing groups will have read the graphing instructions (see below) and went through the exercise to create a graph before starting the experiment.

\section{Graphing instructions}

The following are the steps required to create a graphical representation of the understanding of the text material.

Step 1:

For each section of the material, read the text and select the key concept(s) for understanding the meaning of the text. List these concepts.

Step 2:

For each listed concept in the previous step, draw a node with the concept name inside the node. Do not draw duplicate nodes.

Step 3:

For each section in the text, use a link between nodes to indicate if there is a relationship between the concepts as described in that section.

Step 4:

Try to link concepts between the different sections of the text based on your understanding of the text material as a whole.

All participants were asked to return to the experimental site after $48 \mathrm{~h}$ without knowing the reason behind it and were required to take a retention test.

There are two experimental designs that were implemented in this research, the first one is 3 between subject and 1 within subject mixed design, and the second one is 2 between subject and 1 within subject mixed design. They are summarized as follow.

The first design consists of three between groups factors with the last factor used as a blocking factor: Factor $A$ with two levels "Without Graph" (W/G) and "With Graph" (WG); Factor B with three levels "Top-Down" (TD), "BottomUp" (BU), and "Unorganized" (UR); and Factor $C$ with two levels "Low 
Motivation" (LM) and "High Motivation" (HM). The Motivation scores will be used as a blocking factor to identify low from high motivation using values separated by the median score. In each of the six groups, six participants from the set of the lowest motivation scores will be grouped in a "LM" block, while six participants from the set of the highest motivation scores will be grouped in a "HM" block. As a result, there will be 36 participants with "Low Motivation" and 36 participants with "High Motivation" distributed across all six conditions. Consequently, this experimental design results in twelve conditions (see the following graph).

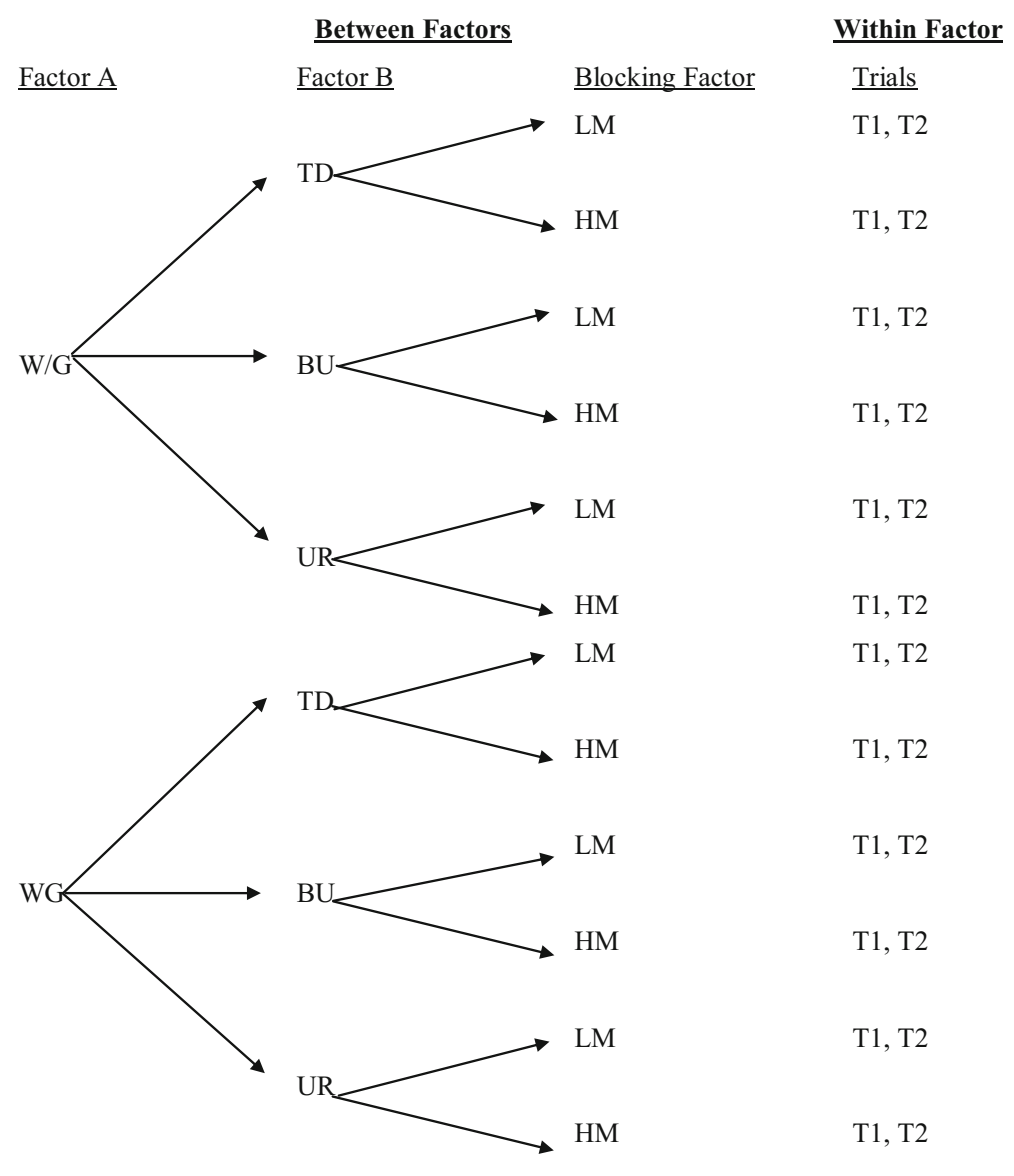

The second design consists of two between group factors: Factor $A$ and Factor $B$ (already described in the above paragraph). The difference between the two designs is that Factor $C$ (Motivation) may need to be eliminated from the design in case it is statistically insignificant. Consequently, this design experiment results in six conditions (see the following graph). 


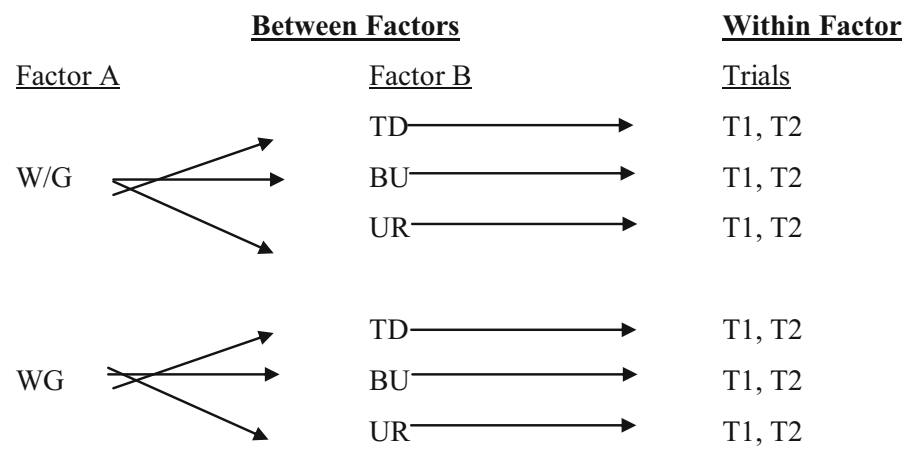

The experimental sessions consisted of two phases. The first phase was involved participant screening, orientation, and was required to take a pretest (see Appendix 1). The purpose of this phase was to gather information on the participants and provide them with an overview of the experiment. A pretest was also given to all participants to assess the homogeneity of participants entering the experiment. A posttest utilizing the same test as the pretest was given following each trial.

The second phase of the study involved experimental trials, each lasting approximately $30-45 \mathrm{~min}$. The instructions for the participants were the same for each trial and each test. Participants were allowed to study the presentation at their own pace and had an unlimited amount of time for studying the material. When they finished the reading session, the participants in the control group were directed to a posttest, while the participants in the experimental groups were provided with a paper and pencil to draw a graphical representation of their understanding of the content they had just read. After completion of the drawing phase, the participants in the experimental group were directed to take a posttest. Upon completion of the posttest, trial 1 is terminated, and participants again were asked to re-open the instructional system for the appropriate presentation. Upon completion of the posttest in trial 2, participants were told they had done with the experiment. During the test, participants did not have access to the training material. The test was the same test for each trial. The measures of performances that were assessed during the experiment were pretest score, posttest score 1, posttest score 2, Retention test score, number of nodes (for the graphing groups only), and number of links N1 (for the graphing groups only). The following is a representation of the different phases of the experiment in a timeline style:

Participant screening --------> Orientation -------> Pretest --------> Reading Material ------> Draw Graphs if experimental group, otherwise move to next step ---> Posttest 1 --------> (End of Trial 1) -------> Reading Material ---------> Draw Graphs if experimental group, otherwise move to next step -----> Posttest 2 ------> (End of Trial 2) -----> Retention Test after $48 \mathrm{~h} \mathrm{----->} \mathrm{(End} \mathrm{of}$ Experiment). 


\section{Results}

An ANOVA was performed to establish the equality of all the groups' participants (see ANOVA results table below). The results indicate that the pretest for all groups was not significantly different. The majority of subjects did not answer any questions in the pretest. This was expected due to the unfamiliarity with the curriculum and consequently, the test.

To determine the effects of graphing, organization, motivation levels and trials on test scores, an ANOVA test was conducted, which resulted in a main effect for the organization factor $(B), F(2.60)=7.09, p=0.002$, beta $=0.082$ (see ANOVA results table below).

ANOVA results on pretest

\begin{tabular}{lrrrrrr}
\hline Source & \multicolumn{1}{l}{ SS } & df & MS & $F$ & Sig. & $\beta$ \\
\hline Total & 33.330 & 71 & & & & \\
Graphing & 0.281 & 1 & 0.281 & 0.568 & 0.454 & 0.885 \\
Organization & 0.215 & 2 & 0.108 & 0.217 & 0.805 & 0.918 \\
Motivation & 0.087 & 1 & 0.087 & 0.175 & 0.677 & 0.930 \\
Graphing $\times$ organization & 0.188 & 2 & 0.094 & 0.189 & 0.828 & 0.922 \\
Graphing $\times$ motivation & 0.781 & 1 & 0.781 & 1.578 & 0.214 & 0.765 \\
Organization $\times$ motivation & 0.049 & 2 & 0.024 & 0.049 & 0.952 & 0.943 \\
Graphing $\times$ org. $\times$ motivation & 2.021 & 2 & 1.010 & 2.041 & 0.139 & 0.595 \\
Error & 29.708 & 60 & 0.495 & & & \\
\hline
\end{tabular}

ANOVA results on test score for trial 1 and 2

\begin{tabular}{lcccccr}
\hline Source & SS & df & MS & $F$ & Sig. & $\beta$ \\
\hline Total & 2982.6 & 143 & & & & \\
Between subjects & 1945.2 & 71 & & & & \\
Graphing & 0.465 & 1 & 0.465 & 0.020 & 0.887 & 0.94 \\
Organization & 325.2 & 2 & 162.6 & 7.09 & 0.002 & 0.08 \\
Motivation & 18.6 & 1 & 18.69 & 0.815 & 0.370 & 0.85 \\
Graphing $\times$ organization & 56.03 & 2 & 28.01 & 1.22 & 0.302 & 0.74 \\
Graphing $\times$ motivation & 0.032 & 1 & 0.032 & 0.001 & 0.970 & 0.95 \\
Organization $\times$ motivation & 140.03 & 2 & 70.01 & 3.05 & 0.055 & 0.43 \\
Graphing $\times$ org. $\times$ motivation & 28.49 & 2 & 14.24 & 0.621 & 0.541 & 0.85 \\
Error & 1376.2 & 60 & 22.93 & & & \\
Within subjects & 1037.3 & 72 & & & & \\
Trials & 763.23 & 1 & 763.2 & 187 & 0.000 & 0.00 \\
Trials $\times$ graphing & 3.204 & 1 & 3.204 & 0.786 & 0.379 & 0.85 \\
Trials $\times$ organization & 9.040 & 2 & 4.520 & 1.10 & 0.337 & 0.76 \\
Trials $\times$ motivation & 4.995 & 1 & 4.995 & 1.22 & 0.273 & 0.80 \\
Trials $\times$ graphing $\times$ organization & 2.731 & 2 & 1.365 & 0.335 & 0.717 & 0.89 \\
Trials $\times$ graphing $\times$ motivation & 0.083 & 1 & 0.083 & 0.020 & 0.887 & 0.94 \\
\hline
\end{tabular}




\begin{tabular}{lcrllll}
\hline Source & SS & df & MS & $F$ & Sig. & $\beta$ \\
\hline Trials $\times$ org. $\times$ motivation & 5.559 & 2 & 2.780 & 0.682 & 0.510 & 0.84 \\
Trials $\times$ graphing $\times$ org. $\times$ motivation & 3.939 & 2 & 1.969 & 0.483 & 0.619 & 0.87 \\
Error & 244.59 & 60 & 4.077 & & & \\
\hline
\end{tabular}

Further post hoc analysis of test scores using the Tukey Honestly Significant Difference test (Tukey HSD) revealed that the bottom-up organization outperformed the top-down and the Unorganized groups on mean test scores across the two trials (see table below).

Tukey pairwise comparison for the organization effect

\begin{tabular}{llcll}
\hline (I) organization & (J) organization & Mean difference (I-J) & SE & Sig. \\
\hline Bottom up & Top down & $2.4760^{*}$ & 0.97761 & 0.037 \\
& Unorganized & $3.5973^{*}$ & 0.97761 & 0.001 \\
Top down & Bottom up & $-2.4760^{*}$ & 0.97761 & 0.037 \\
& Unorganized & 1.1213 & 0.97761 & 0.489 \\
Unorganized & Bottom up & $-3.5973^{*}$ & 0.97761 & 0.001 \\
& Top down & -1.1213 & 0.97761 & 0.489 \\
\hline
\end{tabular}

The effect of Motivation was found to be insignificant and therefore was removed for follow on analysis. The outcome of the ANOVA with motivation removed yielded similar result for the factors graphing, organization, and trials, as was the case, for the ANOVA incorporating the motivation factor.

As for score gain, these was no finding of any factor, either graphing or organization, being significant. As for Retention, there were no main and interaction effects as a result of the ANOVA analysis. There were also no main and interaction effects as a result of the analysis on the number of nodes or the links.

\section{Discussion}

The experimental results show in general that the Organization factor had the greatest impact on learning abstract knowledge as presented in this experiment, with the bottom-up approach having the most positive effect relative to top-down and unorganized conditions.

Motivation is typically an uncontrolled factor in computer-based instruction experiments. In this experiment, however, it was introduced to control for any discriminable differences detected between subjects. The first analysis was conducted with Motivation as a factor, and it was determined that it was not a significant factor. Because motivation was insignificant, it was removed from the analysis, and only graphing, organization, and trials were employed to determine any significance in terms of main and interaction effects. It also increased the degrees of freedom for error terms to increase the power of the $\mathrm{F}$ test conducted. 
Organization was found to be an important factor in learning Industrial Engineering abstract knowledge. This factor had three levels: Bottom-Up, Top-Down, and Unorganized. It was found that the Bottom-Up group outperformed every other group in all measures involving test score performance. This provided a strong indication that the Bottom-Up organization is most suitable for Industrial Engineering abstract knowledge. On the other hand, neither the Top-Down nor the unorganized levels were significant in the learning of abstract knowledge. There was no difference between these two organization levels on their contribution to learning Industrial Engineering abstract knowledge relative to the Bottom-Up level. It is surprising that even with some kind of organization in the Top-Down level, students still did not outperform the Unorganized group.

Consistent with Gagne and Briggs (1974), the Bottom-Up level seems the best content organization in terms of learning Industrial Engineering abstract knowledge as well as other forms of STEM type knowledge that are based on abstract as opposed to physical aspect. However, Williams et al. (in progress) have found the Top-Down model promotes better learning when the content relates to task knowledge (how-to-do-it) and physical system knowledge (how-it-works) contrary to the Gagne and Briggs (1974) position. Similarly, Simon (1981) and Miyake (1986) also identified that a Top-Down organization of knowledge is evident when individuals report their understanding of physical information. The findings herein, on the other hand, do support the Gagne and Briggs (1974) position when acquiring abstract knowledge as it relates to Industrial Engineering. Finally, no differences were found between the different organization levels on retention as measured by the difference between the last posttest scores, and the retention test.

As a result of this experimentation, it was found that graphing of Industrial Engineering abstract knowledge was not an important factor in the learning process. This comes as a surprise because in the graphing group, students created graphs that lay out their understanding of the subject matter requiring greater cognitive activity which in turn should support greater elaboration in depth. No differences were found between any of the organization conditions when graphing was involved. However, there was an increase in number of nodes (i.e., elaboration) and number of links (i.e., depth) between trials consistent with previous findings reported by Chi and Koeske (1983). This finding is explained by the fact that novices would find it difficult to identify relationships between the elements of the organization of abstract knowledge because it is not easy to relate to. Experts in the domain in question, on-the other hand, may easily identify the organization of knowledge and take advantage of the graphing method when learning new knowledge related to the same domain. Anderson et al. (1993) have identified that novices have difficulty understanding the logical relationships among units of knowledge, which is the core of constructing a meaningful graph. By having difficulty understanding relationships, novice students may have difficulties creating graphs and therefore do not benefit from the intended use of the graphing method. Halford (2005) argued that novices unnecessarily increase the cognitive processing load, when trying to segment a complex task. In contrast, experts use conceptual chuncking, which is a form of hierarchical decomposition of knowledge into compressed chunks that reduces the processing load. The graphing method requires the students to segment the content, which, based on Halford (2005), unnecessarily increases their cognitive workload. This may explain why graphing did not improve abstract knowledge learning in these novice students. Furthermore, Stokes et al. (1997) identified that more connections are formed 
and stronger ties are created in the knowledge base when a novice moves through the learning process, when acquiring experience to become an expert. This identified a difference in creating relationships in the organization of knowledge between novices and experts, and therefore, graphing abstract knowledge may be difficult for the novice, and as a result may not fully benefit from it. On the other hand, the literature suggests that experts may be good candidates for the graphing method, when adding new knowledge in their domain of expertise.

This research presents strong evidence that when novice students are presented with an abstract curriculum as it relates to an introduction to Industrial Engineering, they learn better in terms of test performance when the curriculum progresses from the more detailed to the more general. In conclusion, the results suggest that abstract knowledge similar to Industrial Engineering content used in this experiment and STEM type of knowledge be presented in a Bottom-Up hierarchical organization to facilitate the learning process.

In order to understand how these finding can be implemented in real-world education problems, we will use an online intelligent tutoring software example, where student can login and learn different subjects without the intervention of an instructor. The software can be designed to automatically load different organizational structure based on the type of content. For content that is similar to the content presented in this research, the software will present it in a bottom-up approach, while other type of content can be presented in top-down approach. The same software can load different organization of the content to personalize the learning to different students. For example, if a student has demonstrated a learning behavior that suggests his content needs to be presented in a bottom-up fashion, the intelligent tutoring software will present the content in a bottom-up approach, while if another student has demonstrated a different behavior, which would suggest his content to be presented in top-down fashion, the intelligent tutoring software will present him the content in a top-down approach. Therefore, the curriculum can be created once but presented in different hierarchies to meet the different students' needs.

As for future research, and to create a better understanding, we suggest to add a group of novices and have them learn the content in a Bottom-Up fashion. Once they have learned it, the students would be required to report their understanding of the content to determine if they report it in a Top-Down fashion. If so, this would be consistent with how experts report their understanding of a domain as suggested by Shavelson (1972) and Miyake (1986). This is very critical because in the online learning environment example, presented earlier, the intelligent tutoring software can be designed to present the content to a student in different structures at different times based on where they are on the learning curve. A bottom-up hierarchy of the content can be presented to the online students during the first phases of the learning, and as they pass some level of understanding, the curriculum structure can be reversed to a top-down hierarchy to match their expertise level. This new capability will allow the learning software to dynamically change the organization from bottom up to top down as needed to efficiently teach the subject.

As another suggestion, it is proposed to add another condition which would consist of providing the novices with a prepared graph to assist them in forming relationships as opposed to requiring them to create their own graph. This would take the processing load off of the novices to determine if a graph would assist students in the learning process. In our intelligent online tutoring example, discussed previously, the software can be designed to present the student during an online lesson with a graphical 
representation of the knowledge using shapes and links. This new tutoring technology would greatly enhance the learning experience of a student by using the right technology for the right situation and the right student.

\section{Appendix 1: Test and answers}

\section{Test}

Please fill in the blanks in the following questions:

1. (Points: 1)

There are three primary skill groups that a successful industrial engineer (IE) must master: technical, interpersonal, and

2. (Points: 1)

decision making relates to decisions which are biased by the manager's personal feelings or prejudices rather than intellect.

\section{3. (Points: 1)}

The line organization did not always view the industrial engineer as on "their" operations.

4. (Points: 1)

- (For questions 4, 5, and 6) One way to view the professional development of the industrial engineer is to see it as a sequence of three stages:

The first stage is stage.

\section{5. (Points: 1)}

The second stage is the stage.

6. (Points: 1)

The third stage is the stage.

\section{7.(Points: 1 )}

During the stage the IE is learning how to deal with clients, how to design change processes which will involve clients actively in the creation of new methods and systems. 
8. (Points: 1)

Actually showing the client that one's recommendations will work as promised is the definition of

\section{9. (Points: 1)}

In the __ stage, the IE focuses upon learning tools, techniques, methods, mathematical skills, experimental skills, and basic engineering concepts.

10. (Points: 1)

Worker productivity and/or efficiency involves doing things well in the shortest possible time. It requires equipment, tools, training, and

\section{1. (Points: 1)}

labor Employees may not want to admit that the method they were using was not the best, because they will typically resist any change.

\section{2. (Points: 1)}

Overhead labor consists of the costs associated with those individuals involved in making a specific product.

\section{3. (Points: 1)}

One of the roles of the IE to assist clients is to identify, evaluate, accept and then embrace improvement.

\section{4. (Points: 1)}

One of the roles of the IE is to be familiar with line operations prior to the stage.

15. (Points: 1)

- (For questions 15, 16, 17, and 18) Morris has identified four strategies which have been used to promote client change: promised. is actually showing the client that one's recommendations will work as

16. (Points: 1) power of top management to order that it occur. 
17. (Points: 1 )

and is telling the client what advantages are to be found in the

proposed changed or in the new system.

18. (Points: 1)

and promote acceptance which is enhanced by making those whose behavior will be influenced by changes to participate in the planning and design of those changes.

Answer Key (Not part of the test)

\begin{tabular}{|l|l|l|}
\hline Question & Answer & Scoring Matrix \\
\hline 1 & Consulting & 1 \\
\hline 2 & Ad hominem & 1 \\
\hline 3 & Expert & 1 \\
\hline 4 & Analytical & 1 \\
\hline 5 & Problem Solving & 1 \\
\hline 6 & Client Centered & 1 \\
\hline 7 & Client Centered & 1 \\
\hline 8 & Demonstration & 1 \\
\hline 9 & Analytical & 1 \\
\hline 10 & Worker Motivation & 1 \\
\hline 11 & Direct & 1 \\
\hline 12 & Indirectly & 1 \\
\hline 13 & Productivity & 1 \\
\hline 14 & Problem Solving & 1 \\
\hline 15 & Demonstration & 1 \\
\hline 16 & Power, Politics & $1=0.5,2=0.5$ \\
\hline 17 & Selling, Persuasion & $1=0.5,2=0.5$ \\
\hline 18 & Involvement, Participation, Collaboration & $1=0.33,2=0.33,3=0.33$ \\
\hline
\end{tabular}

\section{References}

Anderson, J. R. (1993). Knowledge representation. Rules of the mind. Hillsdale, NJ: Lawrence Erlbaum Associates.

Anderson, J. R., Bellezza, F. S., \& Boyle, C. F. (1993). The geometry tutor and skill acquisition. Rules of the mind. Hillsdale, NJ: Lawrence Erlbaum Associates.

Bousfield, W. A. (1953). The occurrence of clustering in the recall of randomly arranged associates. Journal of General Psychology, 49, 229-240.

Bower, G. H., Clark, M. C., Lesgold, A. M., \& Winzenz, D. (1969). Hierarchical retrieval schemes in recall of categorical word lists. Journal of Verbal Learning and Verbal Behavior, 8, 323-343.

Chi, M. T. H., \& Koeske, R. D. (1983). Network representation of a child's dinosaur knowledge. Developmental Psychology, 19, 29-39.

Frase, L. T. (1970). Influence of sentence order and amount of higher level text processing upon reproductive and productive memory. American Educational Research Journal, 7, 307-319.

Frase, L. T. (1975). Prose processing, the psychology of learning and motivation. Stanford, CA: Gordon H. Bower. 
Gagne, R. M., \& Briggs, L. J. (1974). Principles of instructional design. New York: Holt, Rinehart \& Winston.

Halford, G. (2005). Development of thinking. In K. J. Holyoak \& B. G. Morrison (Eds.), Cambridge handbook of thinking and reasoning. New York: Cambridge University Press.

Miller, G. A. (1956). The magical number seven plus or minus two: Some limits in our ability for processing information. Psychological Review, 63, 81-97.

Miyake, N. (1986). Constructive interaction and the iterative process of understanding. Cognitive Science, 10, 151-177.

National Science Board and National Science Foundation (2002), Science and Engineering Indicators 2002.

Novick, L. R., \& Bassock, M. (2005). Problem Solving. In K. Holyoak \& R. G. Morrison (Eds.), The Cambridge handbook of thinking and reasoning (pp. 321-350). Cambridge: Cambridge University Press.

Scheines, R., \& Sieg, W. (1994). Computer environments for proof construction. Interactive Learning Environment, 4(2), 159-169.

Shavelson, R. G. (1972). Some aspects of the correspondence between content structures and cognitive structures in physics instruction. Journal of Educational Psychology, 63, 225-234.

Simon, H. A. (1981). The sciences of the artificial. Cambridge, MA: The MIT Press.

Stokes, A. F., Kemper, K., \& Kite, K. (1997). Aeronautical decision making, cue recognition, and expertise under time pressure. In C. E. Zsambok \& G. Klein (Eds.), Naturalistic decision making. Expertise: Research and applications. Mahwah, NJ: Lawrence Erlbaum.

Williams, K. E., Crumpton-Young, L., Furterer, S., Rabelo, L. \& Alexandar - Snow (In progress). Reengineering the Undergraduate Industrial Engineering curriculum to better prepare future graduates". American Society of Engineering Education.

Williams, K. E., \& Lopez, F. L. (in press). Mental models, maintenance and complex physical systems. Journal of Military Psychology.

Saleh Al-Foraih has obtained his Ph.D. from the University of Central Florida in Orlando (Florida) in the field of Training and Simulation. He has been involved in the field of teaching since 1994 as a computer science professor in King Abdul Aziz Academy, where he created curriculums for the computer science track and taught multiple courses in the department. He has developed multiple web-based instructional systems for his courses. This is where his interest in curriculum organization and its effect on learning has started, as well as computer-based learning. Dr. Saleh is now a Computer Science Professor at King Abdul Aziz Academy.

Kent Williams is an Associate Professor in the Industrial Engineering and Management Systems Department in the College of Engineering and Computer Science at the University of Central Florida. He received a Ph.D. in Cognitive Psychology/Human Information Processing from the University of Connecticut and an M.A. in General Experimental Psychology/Neuroscience from Connecticut College. Dr. Williams's research and teaching interests are Simulation and Training Systems Design and Development, Cognitive Science, Cognitive Simulation and Cognitive Modeling, Intelligent Tutoring Systems, Human Computer Interface Design and Evaluation, Human Learning and Memory, Machine Learning, Data Mining, Advanced Statistical Techniques, Decision Making, Models of Problem Solving, Management Systems Engineering, Decision Support Systems, Marketing, and Management of Technical Research and Development. 